\title{
The temporal organization of conversation while mucking out a sheep stable
}

Leelo Keevallik

\section{Book Chapter}

Cite this chapter as:

Keevallik, L. The temporal organization of conversation while mucking out a sheep stable., In Deppermann, A., Streeck, J . (eds), Time in Embodied Interaction:

Synchronicity and sequentiality of multimodal resources, Amsterdam/ Philadelphia: J ohn Benjamins Publishing Company; 2018, pp. 97-122.

ISBN: 9789027201157 (print), 9789027263773 (online)

DOI: https:// doi.org/ 10.1075/ pbns.293.03kee

Pragmatics \&amp; Beyond New Series, ISSN: 0922-842X, No. 293

Copyright: J ohn Benjamins Publishing Company

The self-archived postprint version of this journal article is available at Linköping University Institutional Repository (DiVA):

http:// urn.kb.se/ resolve?urn=urn:nbn:se:liu:diva-160748 


\section{The temporal organization of conversation while cleaning a sheep stable}

Running head: Conversation while cleaning a sheep stable

Leelo Keevallik

Professor of Language and Culture

Department of Culture and Communication

Linköping University

58183 Linköping

Sweden

leelo.keevallik@liu.se

\section{Abstract}

Based on talk-oriented activities, there seems to be a consensus that turn-taking is organized to minimize gaps between turns. This study looks at a conversational sequence that evolved in a multi-party setting during a joint cleaning of a sheep stable, and analyzes how nextness is accomplished in a nonproblematic manner after extensive silences. It argues that due to the non-cognitive but physically straining nature of the activity in a confined space, chatting is almost constant but response relevance is reduced. It discusses the moral orders of talk and work in this multiactivity setting, where urgency is not an issue, and suggests that data collection for sequence analysis be more attentive to the systematic differences between talk-oriented and other settings.

Keywords: sequencing in conversation, silence, lapses, response relevance, Estonian

\section{Introduction}

We have substantial knowledge about conversational mechanisms and how speakers negotiate opportunities to speak according to the basic one-at-a-time organization. This paper studies a setting where the auditory space is often wide open for any present party to take a turn, while all the potential speakers are simultaneously involved in the heavy physical task of cleaning a sheep stable. It discusses the temporal evolvement of conversational sequences, the different embodied ways that talk can be designed "for the stage" or as only to be overheard, as well as the variable moral obligations related to the two relevant participation frameworks: talk and non-cognitive work. It will be shown that the sequencing of social actions, and in particular the occurrence of silence, is considerably affected by the dual engagement of the participants, which raises issues about our data collection practices and their consequentiality in terms of the systematicity we discover. The paper also begins to explore the question, whether and how it matters for social interaction that talk is produced with speech organs in the settings where the body itself is under strain.

Conversational turns are generally produced with no gap and no overlap (Sacks et al. 1974) so that silence between turns is minimized (Stivers et. al 2009). There are, however, states of "open state of talk" (Goffman 1981:134) or "incipient talk" (Schegloff 
2007), when people are co-present and speakers move between engaging in, and disengaging from, talk. This has been studied during group work at school, where questions, noticings and outlouds can be deployed as methods of re-engagement (Szymanski 1999). The systematic arrival at, and organization of, lapses have recently been analyzed by Hoey (2015). He discusses cases of relevant cessation of talk at sequence closure or due to the participants attending to some now-relevant contingency, as well as cases of conspicuous absence of talk. Some of his data come from settings where participants are oriented to the optionality of talk: at the occurrence of silence the speakers were going on doing what they were already doing, such as watching TV (Hoey 2015: 437-442). The current study, from a somewhat similar setting featuring optional talk, builds on these findings but will instead focus on how conversational sequences are nevertheless developed in an extended co-presence across longer segments of time.

Silence in itself has been of great interest in cultural studies, where there are several more or less anecdotal accounts available for exceptionally short and long pausing (Lehtonen and Sajavaara 1985, Tannen 1985, Sajavaara and Lehtonen 1997). In closer analysis of conversational sequences, it has been argued that a lapse in talk is simply a byproduct of the recognizable completion of a course of action, be it a projected sequence or a sequence of post-expansions (Szymanski 1999: 14; one type in Hoey 2015). After a lapse, a topic is likely to change (Sacks et al. 1974). At the same time, actions that do not make relevant a response, or do only weakly so, have also been under scrutiny. Stivers and Rossano (2010) argue that announcements, assessments and noticings have generally low response relevance, even though it can be increased by interrogative grammar, interrogative prosody, gaze on the recipient, and recipient-tilted epistemics, i.e. that the recipient is more knowledgeable on the topic than the speaker. In any case, there are regular sequential positions in conversation where silence can ensue. The current paper discusses response relevance and silences within a continuously evolving sequence in relation to a simultaneous parallel activity, hard physical labor.

Several early multimodal studies already showed examples of speakers withdrawing from conversation due to alternative activities. Jefferson (1989) described a case when a participant writes down an address, which results in a pause. Goodwin (1981:105) talks about "activity-occupied withdrawal" from the conversation when a participant gets ready to inhale on a cigarette. Language use and embodied behavior has been studied in settings where the speakers engage in multiple activities (e.g. Goodwin 1984, Mondada 2011). A speaker can, for example, display his multiple engagement by posturing his body in a twist (Schegloff 1998), and a teacher can control a student by touch while engaging in an alternative discussion (Cekaite 2015). A recent collection of empirical studies (Haddington et al. 2014a) has systematically targeted the achievement of multiactivity in various contexts and across a number of languages. The main questions of the collection are also crucial for this study: How do multiple activities co-exist? How do participants display, make recognizable and communicate to others their participation and involvement in multiple activities, through their talk and embodied conduct? (Haddington et al. 2014b: 18). In the current setting, the essentially embodied work task and the mostly verbal talk do not necessarily impede each other, as has also been shown in earlier studies: people can separate (parts of) the 
body from their verbal activities (Raymond and Lerner 2014). However, talk is also produced by the body, which can be problematic under heavy strain. While most interaction analytic work considers talk in parallel to activities that are not particularly strenuous for the body, such as watching TV, studying, or driving (e.g. Szymanski 1999, Hoey 2015), the current paper focuses on a particularly strenuous parallel activity that not only smells bad but also wears out the body. This seems to affect the occurrence of silence between speaker turns. The sheep stable situation offers several opportunities to observe the mutual organization of talk and the work task.

\section{The data}

The study is based on a recording of a single whole-day event where eleven young people had to manually clean a sheep stable of dung. According to an age-old Estonian farming tradition, they are offered food and weekend accommodation for helping out with a tedious task. They are thus not hired as laborers. One of the participants, the organizer and host, has cleaned the stable once before and is therefore occasionally treated as an expert to address questions to, but he is not present during the segment used in this chapter. All the others seem to lack prior experience of the job. At their disposal they have two wheelbarrows, and a number of forks and spades. Without a prior master plan, they switch the tools and the related assignments during the day. The video-recording comprises 5.5 hours from inside the stable. All the participants have consented to the materials to be used for research. The names have been replaced in the transcripts.

Co-presence in the sheep stable situation provides many opportunities to be heard, as the space is small and there is little disturbing noise. At the same time, nobody is entitled to non-task-related talk, nor is anybody pre-assigned the role of an audience, as in e.g. classroom. Thus, the opportunities and entitlement to talk have to be locally claimed and speakers always run the risk of not having a recipient at all. Part of the problem is the fact that gaze may be difficult to use, as people are bending down and lifting forkfuls of dung in various positions, not necessarily facing each other. The participants' bodies are rhythmically preoccupied with cleaning and the question is, whether and how this affects the organization of conversation. This paper explores talk that is not related to the work task, even though it is touched off by something in the concrete environment.

\section{Temporally extended sequence: the case of repair}

There are periods of silent work in the data, increasingly so as the time goes by and the workers grow tired. Let us start by looking at a representative segment of talk from a later part of the day, after more than three hours of hard work. During excerpt (1), Vello and Mari are manouvering the two wheelbarrows, rolling in one at a time to be filled. The participants have organized their spatial placement so that the wheelbarrows can be positioned in the middle, while the diggers form a circle around it (as shown in Figure 1). For 39 seconds, no words have been uttered. When Vello arrives with his wheelbarrow, he puts it down, stands up straight in a leisurely manner, and utters line (1). 


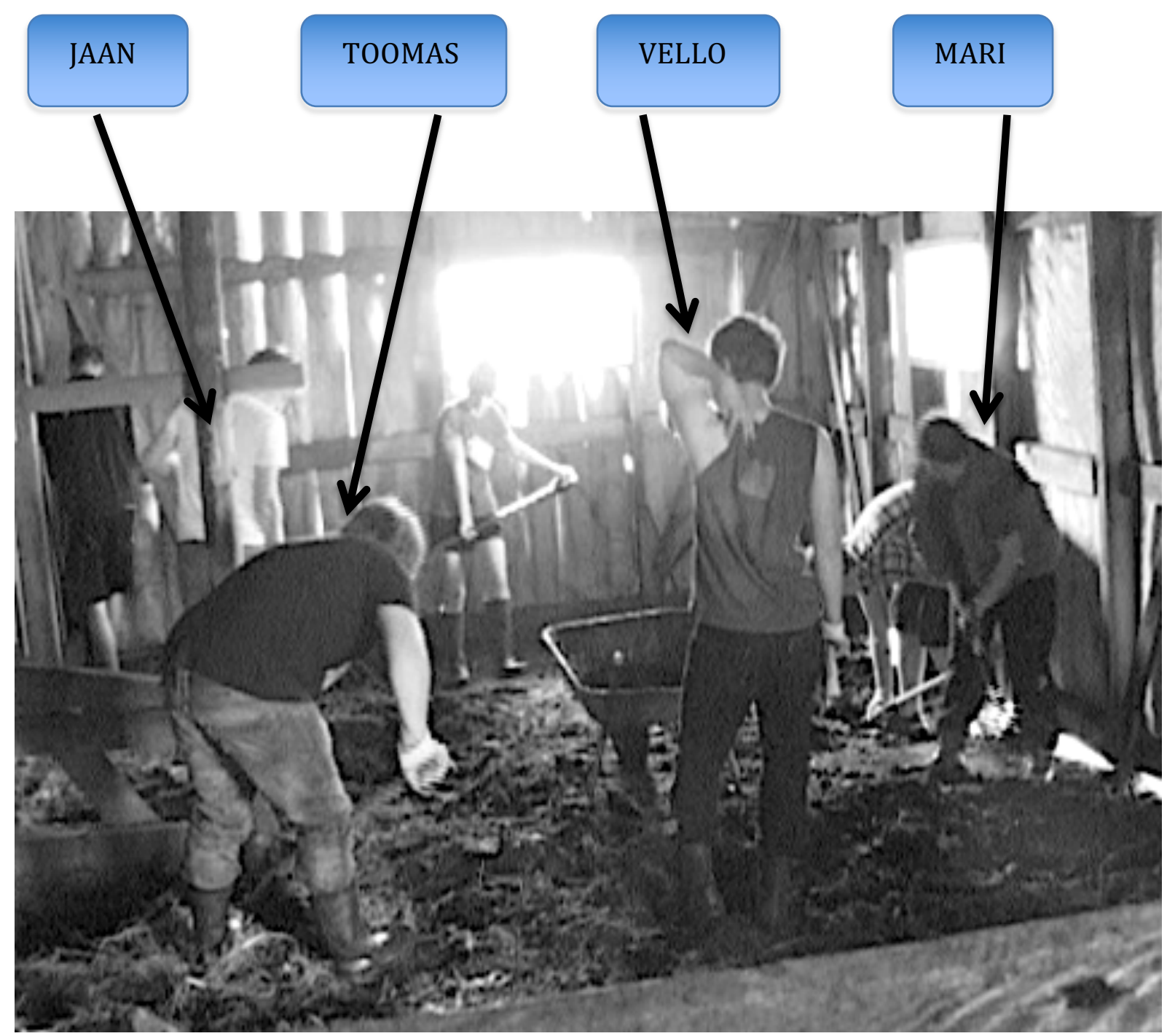

Fig 1: Line (1): Workers from the left: Veiko, Jaan, Toomas (bending over in the front), Piia (lifting), Vello, Siim (hardly visible behind Vello), Rain, Mari. Name labels show the participants who will be involved in the conversation. Vello in leisure position in front of the wheelbarrow, scratching his back.

(1) Herakles I; Sõnnik 5-02-20:35

(39 seconds of silence: Before the beginning of the excerpt Vello has just arrived in the middle with his wheelbarrow, replacing Mari)

1 Vello: vat see \#mulle meenutab seda Eraklese talli jah. VAT this I:ALL remind:3SG this:PRT NAME:GEN stable:PRT yeah 'See, this indeed reminds me of the stables of Herakles.' \#Fig. 1

$2(6.5)$

3 Mari: Au\#gease.

'Augeas'

\#Fig. 2 


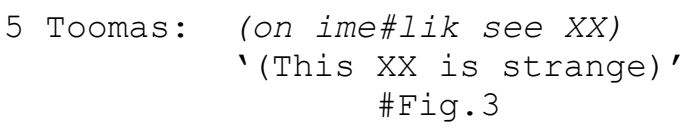

Nobody reacts to Vello's comment for 6.5 seconds. Mari puts a forkful of dung into the wheelbarrow and hunches for a next one. She then turns halfway up, freezing in this position (see Figure 2), and initiates a repair. It is a correction of the reference Vello has used (line 3): the stables in the myth were actually owned by the king Augeas. Nobody reacts to this replacement repair. After a lengthy pause Toomas instead produces a mumbling comment about the surface (line 5).

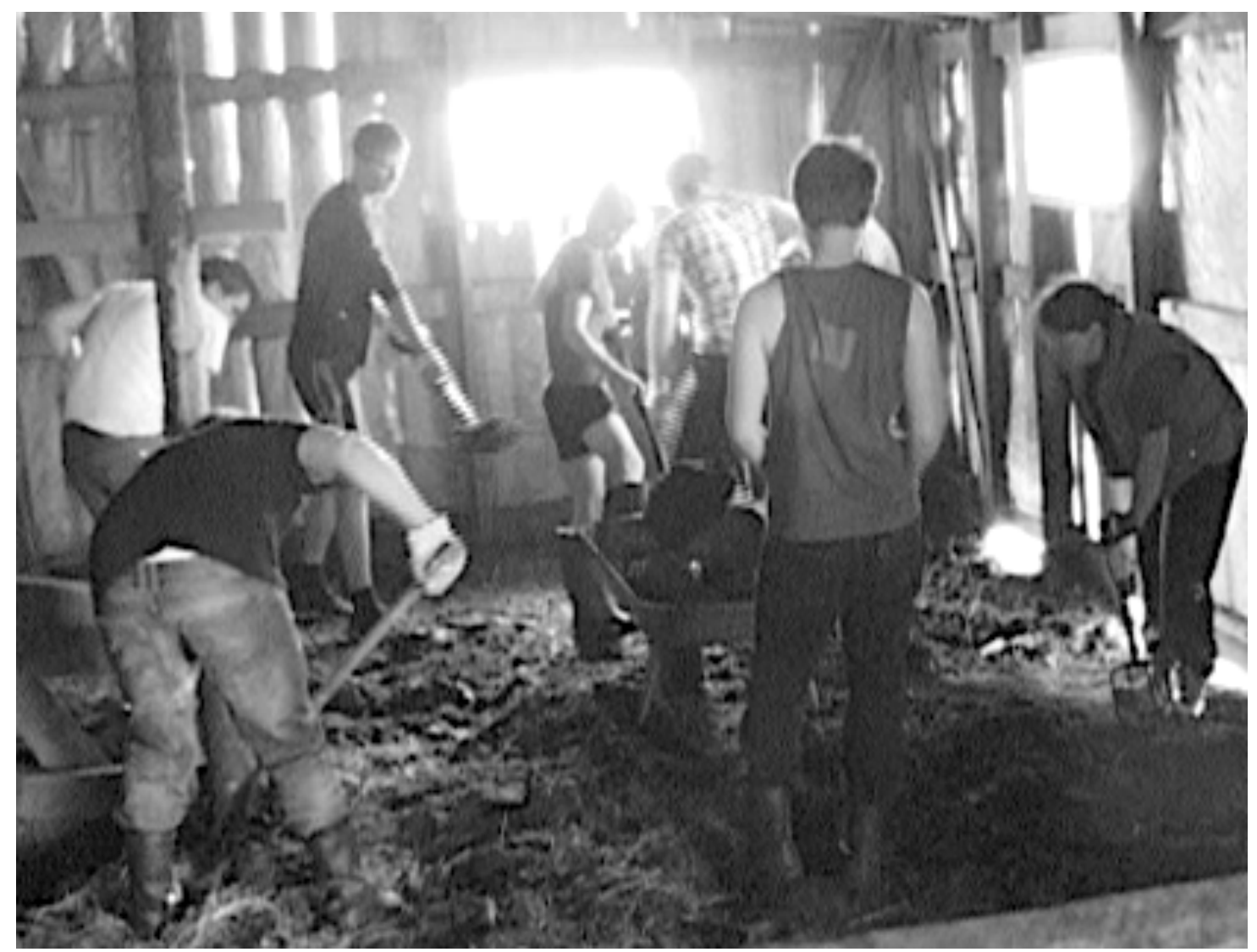

Fig.2. Line (3): Mari halfway up, gaze down, in digging position.

This excerpt is interesting for several reasons: the action sequence, the address, the apparent lack of recipiency, and the timing. Lines (1-3) constitute a repair sequence, one of the central most tightly organized sequences ever disclosed in conversation analysis (Schegloff 2007, Hutchby and Wooffitt 2008, Sidnell 2010). This is an instance of otherinitiation of other-repair, a correction. Indeed, it has been demonstrated that there is a general preference for self-repair in conversation, and that other-initiation of repair may be delayed to provide an opportunity for a self-repair (Schegloff et al. 1977). However, none of the provided examples in Schegloff et al.'s paper come close to a pause of 6.5 seconds that we see in the above excerpt.

At least the following factors contribute to why this temporally distant repair works: 
1. The participants, including the two speakers in the repair sequence, are continuously co-present.

2. Nobody else takes the turn during the silence of 6.5 seconds.

3. The word uttered by Mari is recognizable as a replacement repair through its category (a Greek name of a mythological character who is related to Herakles).

4. The repair-initiation is morphosyntactically fitted to the clause where the trouble source occurred (both Herakles and Augeas are in the genitive case).

5. The repair is non-consequential for the work task.

The latter claim will be elaborated below in relation to the different participation frameworks involved in this setting. It is nevertheless a crucial characteristic of this segment of talk that repairing a fact in a historical myth does not in any way assist in the organization of the simultaneous work. In contrast, if there is a chance that there is some relevance of a repair for the work task, it can be carried out at a quicker pace, as is evidenced in the continuation of the conversation, shown in excerpt (2).

(2) Herakles II; Sõnnik 5-02-20:35

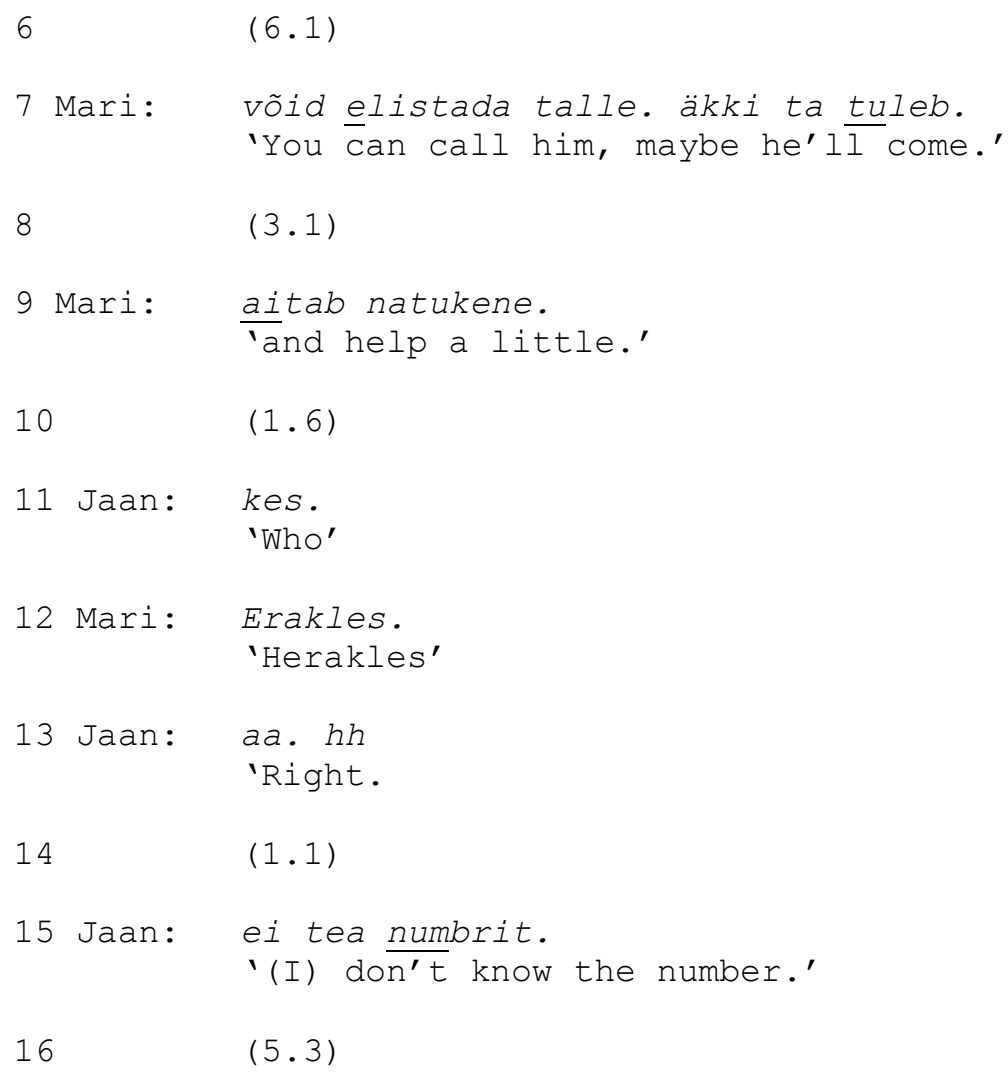

After yet another lengthy silence (in line 6) Mari formulates a suggestion to call Herakles, referentially building on the prior conversation by the use of the pronoun talle 'him'. The pronoun refers to Herakles who was mentioned in line (1) in extract (1), which constructs yet another long-distance link in time. Nobody reacts to Mari's non-serious suggestion and she adds an increment to it, specifying the aim of the call - that he could help out (line 9). At this moment Jaan, who has been working at the opposite side of the room, moves his forkful to the wheelbarrow and initiates a repair: kes 'who?' (line 11). This repair-initiation receives an immediate response, repetition of the name Herakles, and a quick receipt aa 'right' (line 13). Differently from the correction Augease, the 
question kes 'who'? makes a response conditionally relevant, which is one of the reasons that the response is provided immediately. This no-gap production simultaneously demonstrates that a repair sequence with normal pace is in principle possible in this setting, even though the social implications of asking for more information (as in excerpt 2 ) and correcting someone (as in excerpt 1) are different. As to an other-initiation of self-repair ('who?'), there is a social preference for addressing problems as hearing problems first, leaving acceptability implicit (Svennevig 2008), which is why the question receives a repetition of the name as an answer. The repair sequence ends up constituting a textbook example of an insertion sequence between a first pair part, a suggestion, and the account for turning it down in line (15). The pause before the account in line (14) may again reflect the fact that the talk is by now established as being non-consequential for the actual work task.

The repair sequence in lines (11-13) illustrates other important aspects of the activity setting. First of all, it is possible that the physical distance, albeit only a couple of meters, in combination with the sounds of forks, spades, and own heavy breathing occasionally make it difficult to hear. It is more likely, though, that the absorption in work affects awareness of, and attention to, talk. Similarly, the repair initiation in line (11) may reflect the problematicity of keeping track of conversational referents across longer silences. Finally, however, the suggestion in lines $(7,9)$ is about recruiting help, which has more relevance for work, and may thereby attract more attention than a discussion of mythological details.

As shown in excerpt (2) the lengthy silences continue to occur between turns, the most remarkable one still being the 6.5 second pause before the repair initiation in excerpt (1). The original finding of the "no gap - no overlap" organization of talk (Sacks et al. 1974) has found innumerable proofs over several decades of research, a recent crosslinguistic exploration being carried out by Stivers et al. (2009). Stivers et al. have demonstrated that there is a tendency to minimize gaps and overlaps between polar questions and their responses. Whether this means "that turn-taking in informal conversation is universally organized so as to minimize gap and overlap" (Stivers et al. 2009: 10591) remains to be seen. For American English, at least, systematic counts have shown that there is a standard maximum silence of about 1 second between speaker turns (Jefferson 1989). In contrast, Mushin and Gardner (2009) have argued that long silences appear 'ordinary' in Australian Aboriginal Garrwa language community, for up to 13 seconds. They show that "talk may progress with no orientation to the gap and without the gap turning into a conversational lapse. We suggest that this is what is meant by 'comfortable silence'" (Mushin and Gardner 2009: 2049).

In terms of conversational organization, this is exactly what happens in excerpt (1). There is a long silence in line (2) that could easily be a lapse, i.e. a segment of time when conversation has stalled. Mari, by initiating a repair, cancels this interpretation, instead treating it as a regular gap between turns (a similar case can be found in Hoey 2015: 439-440). There are many other gaps in lines (4, 6, 8, 10 and 14), all of them longer than the standard maximum silence found by Jefferson (1989). An obvious question thus arises - is this about culture? Are Estonians equally comfortable with silence as the Garrwa? There is a considerable body of research, more and less precisely documented, that argues for connection between a certain culture and the tolerance of silence (Tannen 1985, Lehtonen and Sajavaara 1985). 
Mushin and Gardner (2009) present several ideas about the features of interaction Garrwa community. They argue, for example, that longer silences can partly be due to the "continuous" nature of communication between the speakers who have known each other all their lives and will continue living close by. Furthermore, Garrwa speakers in their recordings do not have to rush from one place to another according to the clockdetermined schedule. This is clearly different in the current Estonian situation: the participants know each other to some extent, as university students, and they are aiming at getting out of the stable as soon as possible. They are not at leisure and possibilities for speeding up the work is something that they continuously discuss. Excerpt (2) is an illustration of this kind of talk, albeit non-serious. Instead of culture, the current paper would first like to entertain the issue of address (also discussed by Mushin and Gardner 2009) in order to target the question how these temporally extended sequences emerge.

\section{Address and recipiency}

The very first line in extract (1) above, Vat see mulle meenutab seda Eraklese talli jah. 'See, this indeed reminds me of Herakles' stable.', constitutes a noticing action. Noticing has been defined as a comment triggered by something that exists or happens in the context (Sacks 1992:87-97, Bergmann 1990, Schegloff 2007). Schegloff famously analyzed the noticing "You didn't get an ice-cream sandwich" addressed to a specific participant (2007:75) and thus making relevant a response by that particular participant. Likewise, Szymanski (1999) shows that during group work in classroom, noticings require their recipients to look somewhere or do something in order to verify the claim being made, or to resolve the issue being raised (Szymanski 1999: 6). The noticing in line (1), however, is different in that it does not say anything about a specified other. It is generic, and does therefore not make anyone accountable for the complainable matter that their work task is a Herculean one.

In fact, it does not oblige anybody to answer at all. The noticing in line (1) is formulated as a private observation especially by mulle 'to me'. The final jah 'yeah', that approximately corresponds to the English 'indeed' hints at the possibility that there has been prior talk on the topic, but not inevitably. During the utterance Vello alone is standing straight up, while everybody else is digging dung (as shown in Figure 1 above). Vello speaks loud and clear, with his head up and oriented towards the center of the stable, his gaze apparently in mid-distance. His turn is designed to be heard by everybody around him, possibly to offer an opportunity to re-engage in talk (Szymanski 1999). Nobody even lifts their gaze in response.

Toomas' utterance on line (5) on imelik see $X X$ 'this XX is strange' also constitutes a noticing, most probably a comment on the surface, but it is less designed for reengagement by others via his embodied behavior. In contrast to what Vello did, Toomas turns away from the center and all other participants (as shown in Figure 3). He looks down, pokes his fork to the ground, adjusts his glasses, and mumbles the turn. It is difficult to hear exactly what he says but a few minutes earlier he has been complaining about the lack of bottom under the dung - which is how deep they have to dig. Toomas' turn is designed in a much more private way, more as an "outloud" to be overheard, while he himself is inspecting the object of his assessment, the ground. Out-louds are mutterings which leave another free to respond or not (Schegloff 1988, Goffman:117, 
Szymanski 1999), they are self-absorbed and arbitrary in terms of response relevance. In fact, Toomas' comment does attract Vello's gaze- Vello is still waiting for the wheelbarrow to be filled and stands right next to Toomas (Figure 3). However, similarly to Vello's noticing, it one does not result in any response.

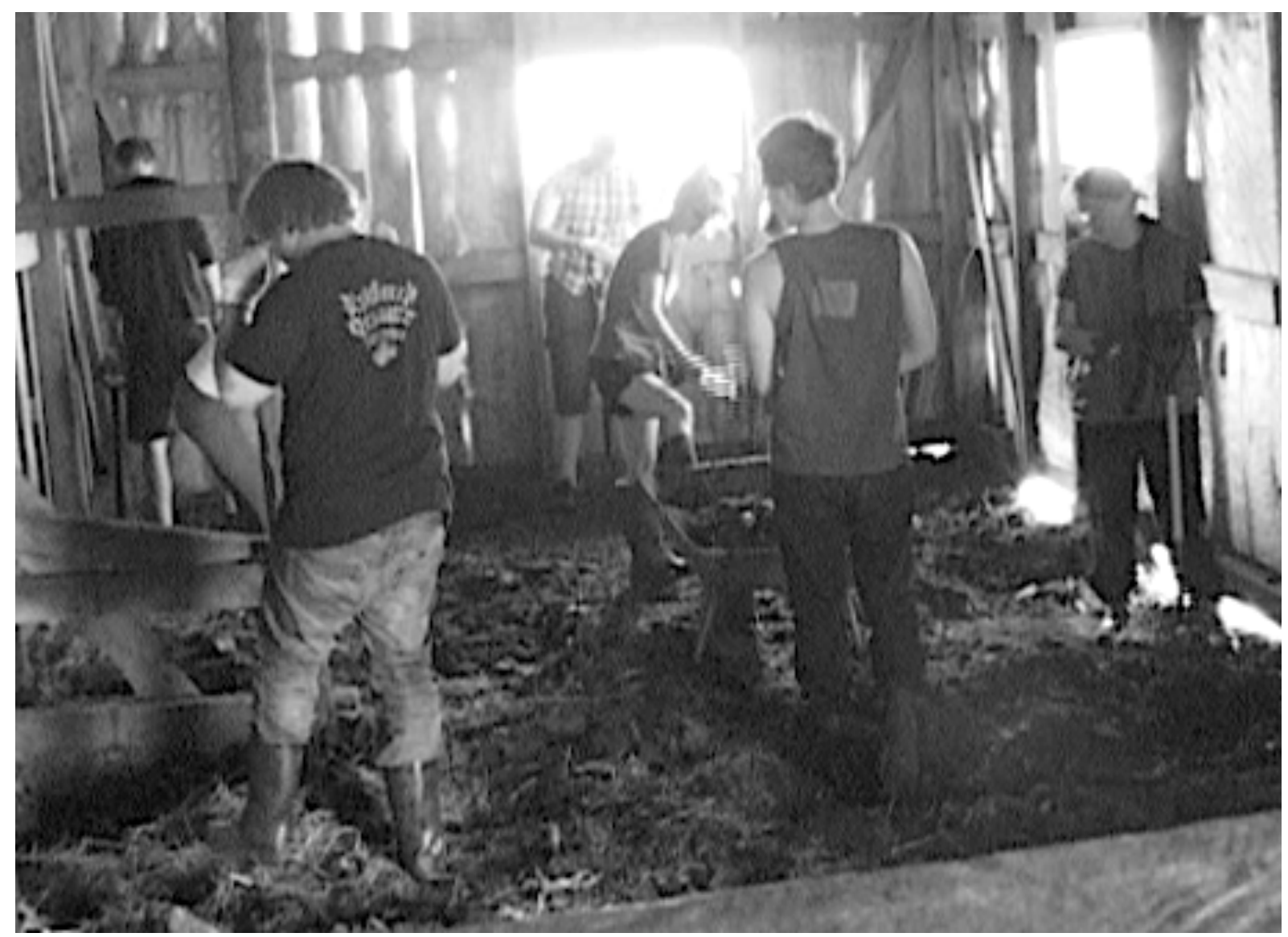

Fig. 3. Line (5): Toomas turning away from the center, looking down, adjusting his glasses, and muttering.

The two noticings (lines 1,5) are dissimilar in their embodied displays, the first one seeking audience, and the other one self-addressed, as is evidenced by its mumbling delivery. None of them singles out a recipient. Relatedly, Mushin and Gardner (2009) found that in situations where "a participant is selected to talk next, silences of more than about $1.5 \mathrm{~s}$ are indeed an indication of trouble". In other words, gaps were shorter when there was explicit next speaker selection, and considerably less problematic when there was none. Also Meyer (2010: 129-154), working on Wolof data, suggests that tolerance of silence has to do with "indeterminacy of addressing and listener roles" (ibid. 153-154). Crucially, actions such as noticings and assessments are among those that only occasionally make relevant a response (Stivers and Rossano 2010), a finding which is supported by the two turns discussed above.

In the above excerpts, however, we also have turns that do select a next speaker but do not necessarily receive a response. A repair-initiation, such as Augease in line (3) selects the previous speaker Vello to accept the repair, which he does not do. On the other hand, Jaan's kes 'Who' in line (11) selects the prior speaker Mari to accomplish the repair, which she does. While initiating the repair Jaan slightly lifts his head, and possibly even 
looks at Mari, who quickly responds by repeating Herakles. Jaan receives the answer with a brief $a a$, a response token that indicates understanding (Kasterpalu and Hennoste 2016). This repair sequence has been "on time", with no gap between the turns. A clear single addressed recipient, both by virtue of the conversational structure and the embodied display, apparently contributes to the question receiving an answer.

Non-responsiveness in the above sequence concerns actions beyond noticings. The suggestion by Mari in line (7) to call Herakles is explicitly addressed to Vello. It is formulated in $2^{\text {nd }}$ person singular, võid elistada 'you:SG can call' and selects the one who mentioned Herakles as the recipient of this turn as well as the future agent to make the call. It is designed as a next to Vello's initial noticing, and thus creating progressivity based on that. Mari also half-turns toward Vello, who does not respond. Instead, after a long silence of 3.1 seconds, an increment, and another lengthy silence of 1.6 seconds, Jaan initiates a repair, and eventually positions himself as the recipient of the suggestion by turning it down in line (15), joining the jocular key. Having a single addressed recipient for the turn does thus not always result in the person responding. Partly, this may have to do with the non-serious nature of the suggestion, provided for entertainment rather than for practical purposes. Accordingly, a relevant next action here could be laughter, which does not occur either.

One reason for the difficulty of attracting a response, and of re-engaging in talk in general, is the presence of a large group of potential speakers who are addressed generically. They are free to not position themselves as next speakers, or even as recipients of talk. Above, we have seen that speakers can produce actions in systematically different embodied ways, displaying readiness for re-engagement, or not. Prosody, gaze, body orientation, and placement in space can be involved in these displays. Nevertheless, in this activity setting, neither an embodied display nor explicit next speaker selection necessarily result in a response. Furthermore, lack of response is not treated as problematic, and appears to be a part of the social organization of talk. In some cases utterances stand alone between pauses, or merely attract someone's gaze (as line 5), which can be seen as a minimal reaction. In other cases, however, the sequence is nevertheless built further in this temporally extenuated manner, as will be discussed in the ensuing section of the paper.

\section{Sequencing in a state of incipient talk}

The last turn we considered above was line (15), where Jaan rejects the suggestion to call Herakles by claiming to lack his phone number. This is followed by yet another long silence of 5.3 seconds, which could have easily qualified as a lapse, a period when conversational activity has ceased. However, the sequence continues by Vello offering (most probably) a random phone number, thus retrospectively treating Jaan's claim about the lack of phone number as a complaint, and offering a solution to it.

(3) Herakles III; Sõnnik 5-02-20:35

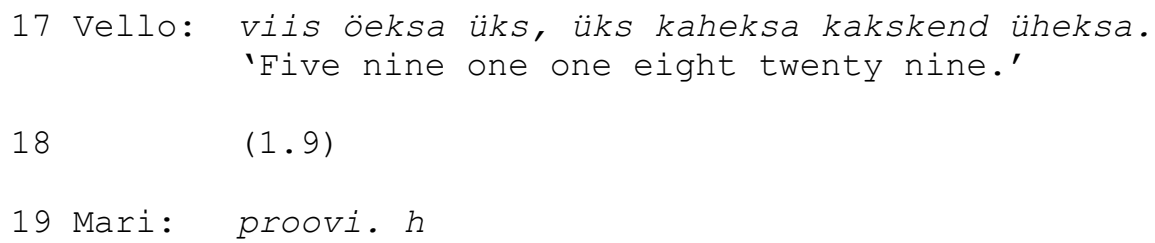




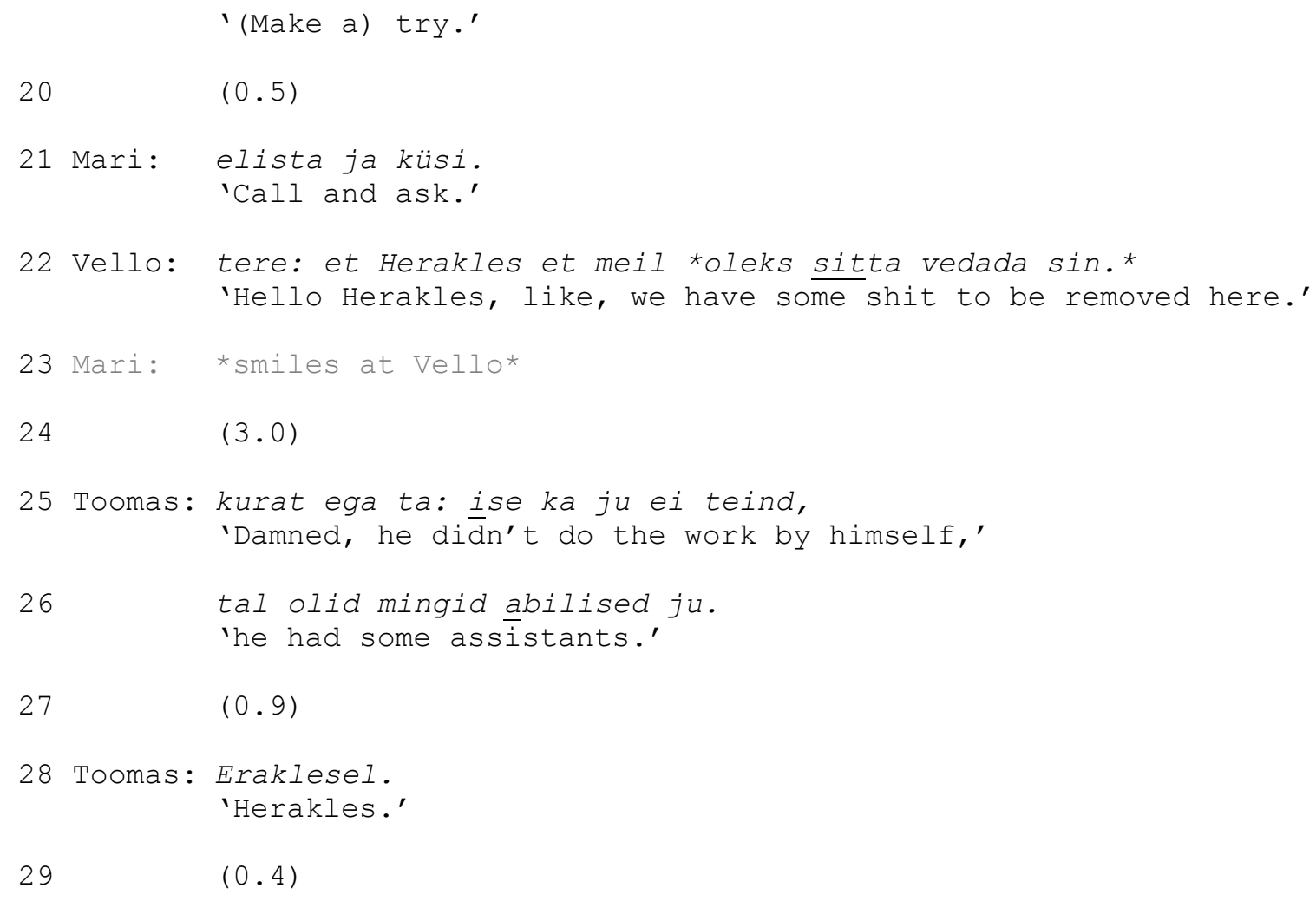

Herakles' phone number (line 17), provided as a response to Jaan's problem formulation, is not clearly addressed to Jaan. Vello's gaze is not visible in the recording but Jaan looks down during and after the turn, which indeed may also be a strategy of displaying nonavailability for the next turn. At the same time, the turn can be heard as an informative announcement by anyone who is co-present.

After a shorter pause (line 18) Mari makes use of the fact that Vello has now positioned himself as possessing the knowledge of how to reach Herakles. She reissues her original suggestion to Vello to make a call, first in a contracted manner. She utters an imperative proovi. '(Make a) try.' in line (19), which structurally builds on her own prior action (võid elistada talle 'you can call him' in line 7). Due to lack of response, Mari expands on the suggestion after a short pause (line 21). After that, instead of literally agreeing to call, Vello produces a possible first line of the phone call. His turn in line (22) could be treated as a punchline of a joking sequence, bringing it to a closure through laughter. Indeed, it does receive a smile from Mari, who also briefly establishes mutual gaze with Vello.

The sequence could as well be abandoned at this moment but after another longer pause Toomas chooses to comment on the whole idea of contacting Herakles by targeting its erroneous grounds - Herakles did not do the job himself. Toomas' turn is formally built on the prior, as it uses pronominal reference (lines 25-26). Albeit an assertion about factual matters, it is designed for response through its grammatical and embodied delivery. Utterance-initial ega is an epistemic stance marker that is regularly used for formatting negative questions in Estonian (Keevallik 2009). Toomas stops working at the beginning of his turn, looks towards the middle of the circle of workers, lifts his head, and speaks out loud. Still, he is not successful in receiving a response, neither at the first completion of his remark, nor at its re-completion in line (28). 
Considering the whole episode presented in excerpts (1-3), it clearly features a sequence, built incrementally and locally, as any sequence in conversation. Schematically, it has consisted of the following actions, with line numbers in parenthesis:

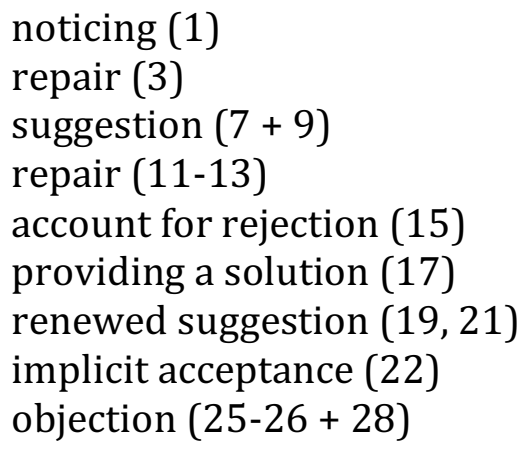

Four different people have participated in the sequence, and contributed to a topically coherent discussion of recruiting help from Herakles. Most of the lines after (1) employ prior talk as a resource, either through pronouns or by being structurally incomplete on their own. The only truly puzzling feature is the unusual silences that never become lapses. The speakers here constantly build further on the sequence, transforming lapses into gaps (as also showed by Hoey 2015). They employ various structural features of language, such as pronouns and incrementing, to construct nextness and achieve progressivity, while silences between turns are not treated as problematic.

The non-serious nature of most of the talk may contribute to the silences. The suggestion to call a mythological character is non-serious, as is the account for not accepting it due to lack of phone number, and finally the mock compliance is performed as a laughable. The first line on the phone to Herkules underlines the totally out-of-place character of the imagined request. This talk is indeed not consequential for anything but participation in talk itself, even when it comes to the more serious matters of correcting mythological facts.

The core interest of conversation analysis has been on ordered sequences, especially adjacency pairs, but there has always awareness of states incipient talk (Goffman 1981, Schegloff 1988, 2007). These states have not attracted major research, possibly because it is only during the last decades that attention has truly shifted from verbal to multimodal interaction (Nevile 2015). Characteristically, also, incipient talk has been studied in many highly complex professional settings where it is produced in relation to the work task, such as surgery, archaeology, or navigating (Goodwin 2002, Mondada 2014). Even in the current sheep stable setting, much talk is about the task itself, about the features of the soil, the tools and the logistics. But since the actual work task is cognitively not demanding, and nothing requires professional attention, there is relative freedom to chat. The auditory space is open and people are co-present, the talk could basically proceed as if at a reception party, but it does not. The potential speakers are bodily preoccupied with a heavy task, which sets a separate moral order for the occasion, as well as essentially appears to affect the temporality of sequencing in talk. 
The overt and primary aim of the recorded event is to clean the stable. The speed of achieving this aim is in direct correlation with how much energy the participants put into the shoveling. This sets the baseline collective moral obligation in the activity setting and each participant is likely to be monitored in regard to their contribution. Overall, participation in the event can imply just doing a good practical job, involvement in physical work being at least as legitimate as participation in talk, if not more so. Beyond task-related instructions and coordination, there is no obligation for the present parties to speak or even listen to talk. At the same time, conversation is governed by a different social and moral order of conversational contingencies and obligations of alignment (Stivers et al. 2011). The two moral orders - for work and for talk - are layered on top of each other during this event, and make relevant different kinds of participation.

In prior literature several terms have been used to describe the relationship between two simultaneous activities, such as main vs. side involvement (Goffman 1963, 1971), prioritised vs. non-prioritised activity (Mondada 2014), and postponable vs. nonpostponable activity. Non-postponable activities may be launched with the help of explicit suspension items, such as hold on (Keisanen, Rauniomaa, \& Haddington 1914). This never happens in the current data, as both activities are in principle postponable. Nevertheless, chatting is sometimes treated as a side-involvement, as illustrated in the following extract, featuring both chatting and task-talk.

At the end of extract (3) Toomas stated a problem with the Herakles-plan. This turn belonged to the domain of chatting, touched off by the local activity but not part of solving the work-task. Almost without a gap, however, Vello utters a next turn (line 30 in excerpt 4), which belongs to the domain of work.

(4) Herakles IV; Sõnnik 5-02-20:35

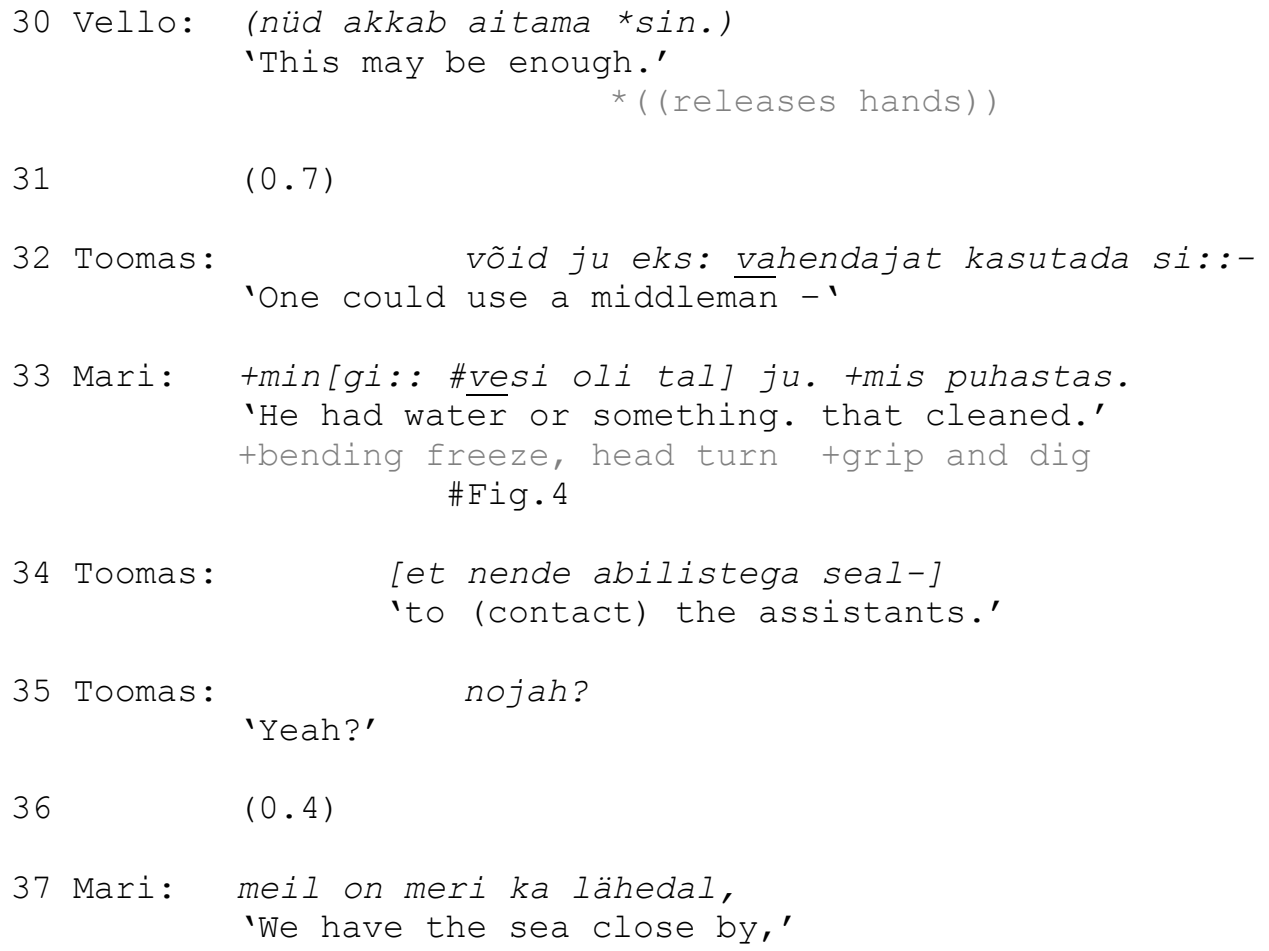


In line (30), Vello comments on the status of his wheelbarrow, that it is now almost sufficiently filled. Wheelbarrows are central for the entire organization of the work task. There are two of them, used for bringing dung outside the stable. The filling and rolling of wheelbarrows determines the work rhythm throughout the day. When they are positioned inside, dung can and should be lifted into them, which results in the diggers being preoccupied. During this period, the manipulator of the wheelbarrow usually takes a rest (as Vello does in extracts 1-3). The job of rolling wheelbarrows is considered the hardest one, and several participants switch to this role during the day. On the other hand, while the wheelbarrow is rolling, the diggers can relax. Accordingly, Vello in line (30) releasing his hands from a resting akimbo position, marks the approach of a major activity junction: the wheelbarrow is about to be full. Throughout lines (31-35) Vello is slowly moving closer to it, and takes hold of the handle on the word meri 'sea' in line (36). This marks the end of the current round of shoveling. The emergence of Vello's turn on the state of the wheelbarrow is not treated as problematic, even though it does not follow up on Toomas' discussion. The work-task oriented turn does not lead to an uptake, and it is also impermeable to the continuation of the Herakles-topic after it.

Similarly, Gardner and Mushin (2007: 13) witnessed situations in their Garrwa data where "talk was not built to be a relevant next", i.e. it was not sensitive to the conversational contingencies. In the current data, this seems to be a systematic feature of talk within the physical task framework: these turns are not necessarily built as relevant next ones in the conversational activity (an example being line 30). Meyer (2010: 147) also hints at the possibility that this kind of building of a turn as not the relevant next may stem from dissimilar participation frameworks. In the above sequence, the very basis of conversation, namely adjacency and nextness (Schegloff 2007: 14-16), seem to be achieved in the chatting sequence regardless of the intervening verbal actions that belong to the different moral order, that of the work task.

Task-related talk is frequently inserted in a non-problematic manner into unrelated conversational sequences, which may demonstrate its priority status. Vello's taskrelated assertion nüd akkab aitama sin, approx. 'This may be enough' (line 30) does not make relevant a verbal response, only an embodied withdrawal by his co-workers. It does not initiate a sequence of talk, and thus does not lead to conversational schisming (as demonstrated in talk-oriented multi-party settings by Egbert 1997). Neither is the assertion particularly urgent, as it still takes several seconds and forkfuls before Vello actually takes the wheelbarrow. It is well-timed with the task but does not require immediate attention, differently from some other non-postponable activities that may take priority over chatting (as illustrated in Keisanen et al. 2014).

Toomas, however, has stopped working by now (since line 25). He leans on his fork and continues talking about using Herakles as a middleman (lines 32, 34), thus taking a morally questionable opportunity to prioritize talk over work. In contrast to Vello who 
has uttered his line (30) relatively low in a routine-like manner, Toomas is here orienting to the center stage, with his head up (as shown in Figure 4), and talking in a loud voice. He thereby designs his turn for recipiency. With his body disengaged from work, he is now only participating in the conversation. In contrast to that, Mari utters her line (33) while simultaneously displaying participation in work (shown in Figure 4). She is bending down, in digging position. During the first half of the utterance mingi vesi oli tal ju. 'He had water or something.' she is frozen in the position, thus not actually doing any work, only displaying continuous readiness for it. She has her head turned towards the middle but gaze on the ground, but she speaks loudly, thus for the audience. During the incrementally added relative clause mis puhastas 'that cleaned', she actually starts digging again. Her gaze is now on the fork and her work area. While Toomas relaxes and projects an audience across a longer sequence of talk, Mari displays orientation to the immediate continuation of work.

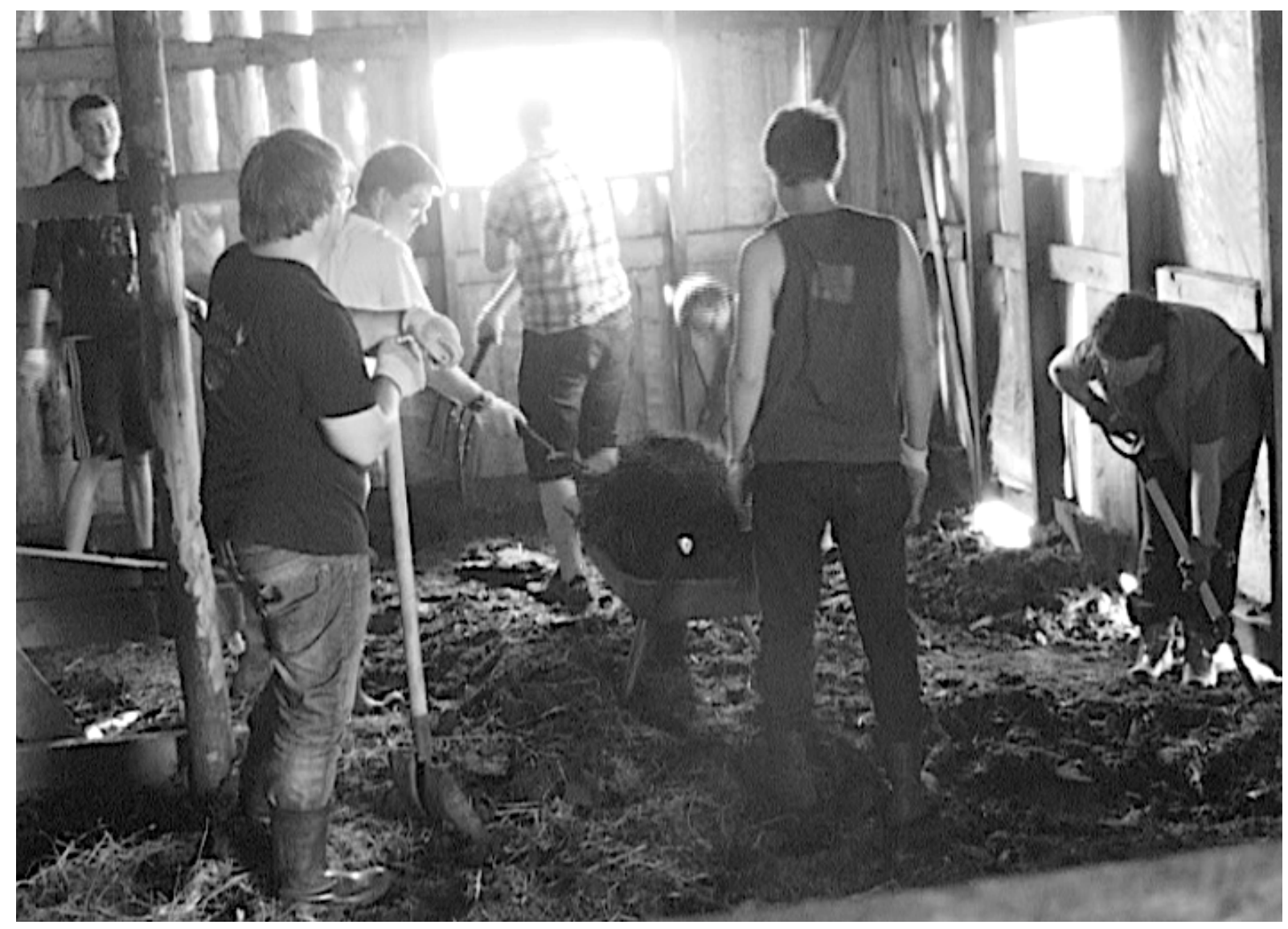

Fig. 4. Line (33): Toomas resting, Mari in digging position.

In this section, we have seen two different kinds of moral order layered on top of each other, as reflected in talk and embodied displays. First, there seems to be a dual order for task-related talk versus free conversation. Utterances that belong to the first order can be produced without orientation to conversational contingencies, displaying their priority in the work-activity framework, and not necessarily disturbing the sequential order of the ongoing conversation. Second, conversational contributions may be accomplished with leisurly composure, or with the body participating in the (displays of) work order. It seems to be possible, at least occasionally, as evidenced in Mari's accomplishment in line (33), to simultaneously satisfy two different social and moral 
orders by deploying vocal resources for conversation, and bodily ones for work. Taskrelated noticings (such as in lines 5,30 ) are timed with the work process, and morally justified by the primary work task. Conversational sequences, in contrast, can be produced independently of work. They are subject to the moral order of contingencies and appropriate next actions, which is established through the design of every turn.

\section{Talk and the body at work}

As it happens, a substantial amount of talk during the day at the stable concerns the work task, the coordination between the participants, and the discussions on what counts as "the bottom" vs. "dung". The latter is crucial in regard to when the job can be defined as done, as is also reflected in the frustrated noticing by Toomas in line (5). In contrast to invitations for re-engagement in conversation (Szymanski 1999) many of the work-task related utterances are not designed for verbal recipiency. At least in the current data, these are instead temporally fitted to the work order and its (too slow) progress. Occasionally they are left with neither verbal nor immediate embodied response of any kind. These responseless actions seem to involve announcements, instructions, outlouds, swearing, and noticings. In the following, short examples of all of these will be provided.

Speaker turns that potentially accomplish an initiating action but are legitimately not responded to are often not designed for anyone in particular but for "everybody" as a single party, or "anybody" who cares to respond. For example, there are general announcements, such as vaba käru vaba käru 'Free wheelbarrow. Free wheelbarrow.' (1 17:31) letting the workers know that another wheelbarrow is arriving, while nobody in particular is expected to work with it at this very moment. Similarly, another participant returns with repaired tools, saying kaks relva tuleb tagasi 'Two weapons coming back.', where 'weapons' refers to work tools (5 02 27:30). This utterance attracts a couple of glances but the tools are subsequently simply placed along the walls. This kind of announcements indeed provide information that at some point may become relevant for the participants, but the local response-relevance is, let alone a verbal one, is low. This conforms to the findings of Stivers and Rossano (2010) who discuss announcements as being low in response relevance (p. 28), especially in case they are produced with noninterrogative grammar and prosody, no gaze, and with speaker-tilted epistemic asymmetry. Except for gaze, all the features are present in the above two examples. Gaze designs these announcements as being addressed to "everybody" (see the section above on address and recipiency), thus constituting a special case of not identifying a single recipient.

Another type of announcements are formulated in first person. These are regularly in the service of formulating one's own actions as a legitimate part of the work task. Examples include ma viin need palgid kuskile välja 'I'll take these poles somewhere outside.' (7 10:30) and ma viin ära 'I'll take (the wheelbarrow) away' (4 13:49). These utterances make the speaker's actions understandable to other co-present parties and only potentially attract a verbal reaction.

Instructions do not need to be verbally responded to either, but they clearly make relevant an embodied compliance, with different temporal horizons (immediate or remote), organizing work. They can address single participants, such as kõige lihtsam oli 
seda serva mingi auk lahti ja sis labidaga tõmbad 'It was easiest to (cut) open a hole from the edge and then you pull with the spade' (5 02 01:40), or everybody who is present võtke siit nüüd pealt nagu vähemaks, sis on sellega kõik ma arvan 'Take a little more here from the top and then I think it'll be all' (9 7:10). These actions of course display an interesting sequential structure of their own, in terms of embodied compliance or noncompliance.

Yet a different group of responseless turns can be described as out-louds (Schegloff 1988 :117). An outloud is a private or semi-public turn designed to be overheard, possibly a muttering that leaves another free to respond or not. A typical case would be a swearing, such as ossa raisk 'damn it' (9 17:45), when the speaker has accidentally hit a co-worker. Swearing is sometimes commented on but not responded to. In another case a speaker specifies the swearing by a realization: mis kurat see on aa, ma astusin sita sisse 'What the hell is this. Oh, I stepped into shit.' (5 02 25:40). This is received with a laughter, a relevant response to something that is taken as a joke. Accounts of reasons that occasioned the swearing can be elaborated in further conversational sequences. Similarly to other out-louds, they can thus potentially initiate sequences.

Noticings comment on things that exist or are happening around the speakers (Sacks 1992:87-97). They actually occasion many sequences and are developed into conversational topics, as we saw in excerpts (1-4). But they may also be ignored, both in the auditory (no answer) and the visual space (no gaze, smile). At one point a participant becomes impatient for not finding the bottom, the soil underneath the dung. He utters numerous turns of the type: kurat see on nagu põhjatu sin, nääd ei jõua põhi kätte noh 'Damned, there is like no bottom here, (we'll) never reach the bottom' (5 0200$)$. These complaints could occasion collective bemoaning, but mostly they do not.

These were some examples of talk that are entirely subsumed to work order and its temporal structure, and where silence can normatively follow. In contrast, in our target episode, the participants orient to two kinds of orders in parallel: talk and physical work. Talk can be produced in the service of the work task as well as of conversation. Likewise, the body may accomplish work as well as contribute to mutual sense-making in conversation. In the following excerpt (5) in lines (39-40), Vello fits his talk to the ongoing discussion on whether they should dig a ditch to carry away dung with a stream (a proposal by Mari at the end of excerpt 4). At the same time he drags the wheelbarrow backwards, out of the stable. His utterance constitutes a disagreement with Mari: in his opinion it is easier to drag away the dung.

(5) Herakles V; Sõnnik 5-02-20:35

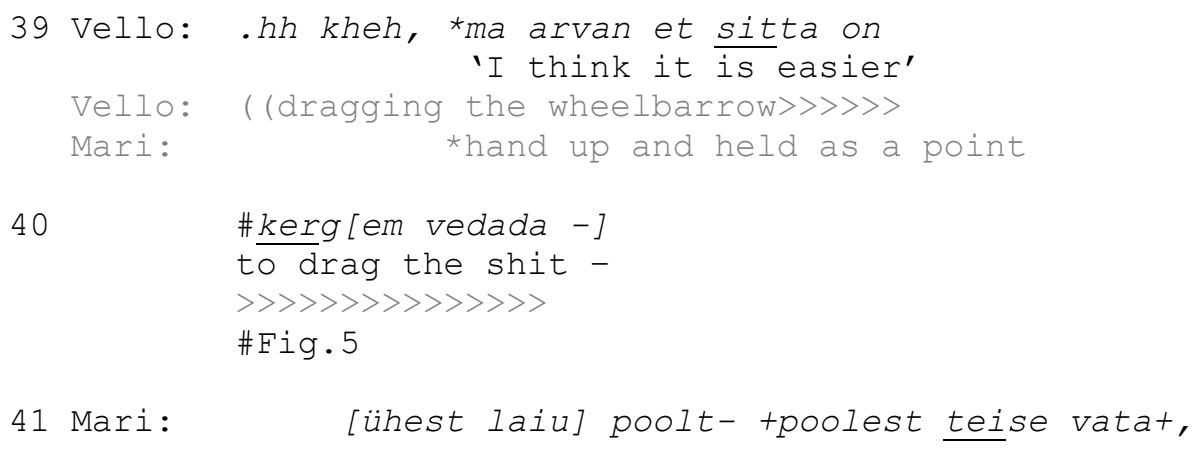




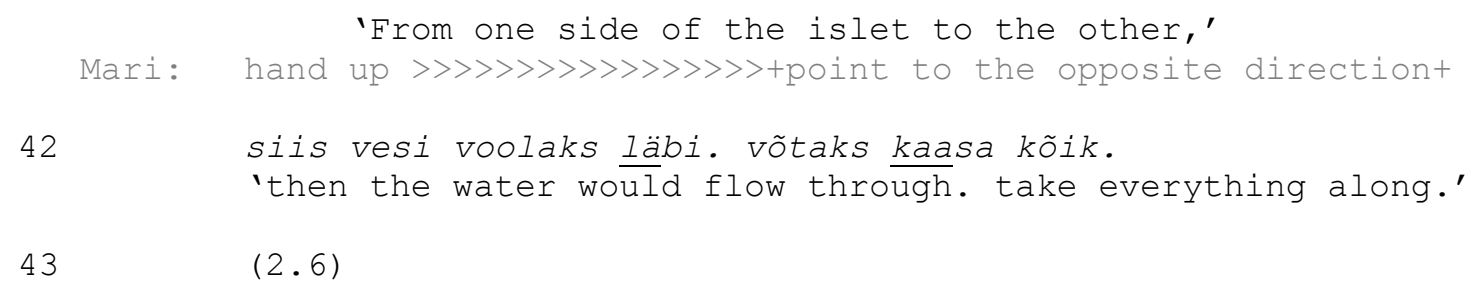

While Vello's body in lines (39-40) is absorbed in the physical task, Mari uses hers for a pointing gesture in line (41), as shown in Figure (5). She is still talking about the ditch and suggesting they dig it from one side of the islet to another. These two ways of using the body illustrate participation in the two different organizations: Vello in work and Mari in conversation. Mari has abandoned work for the time being, she is standing straight up and points out a possible direction of the ditch.

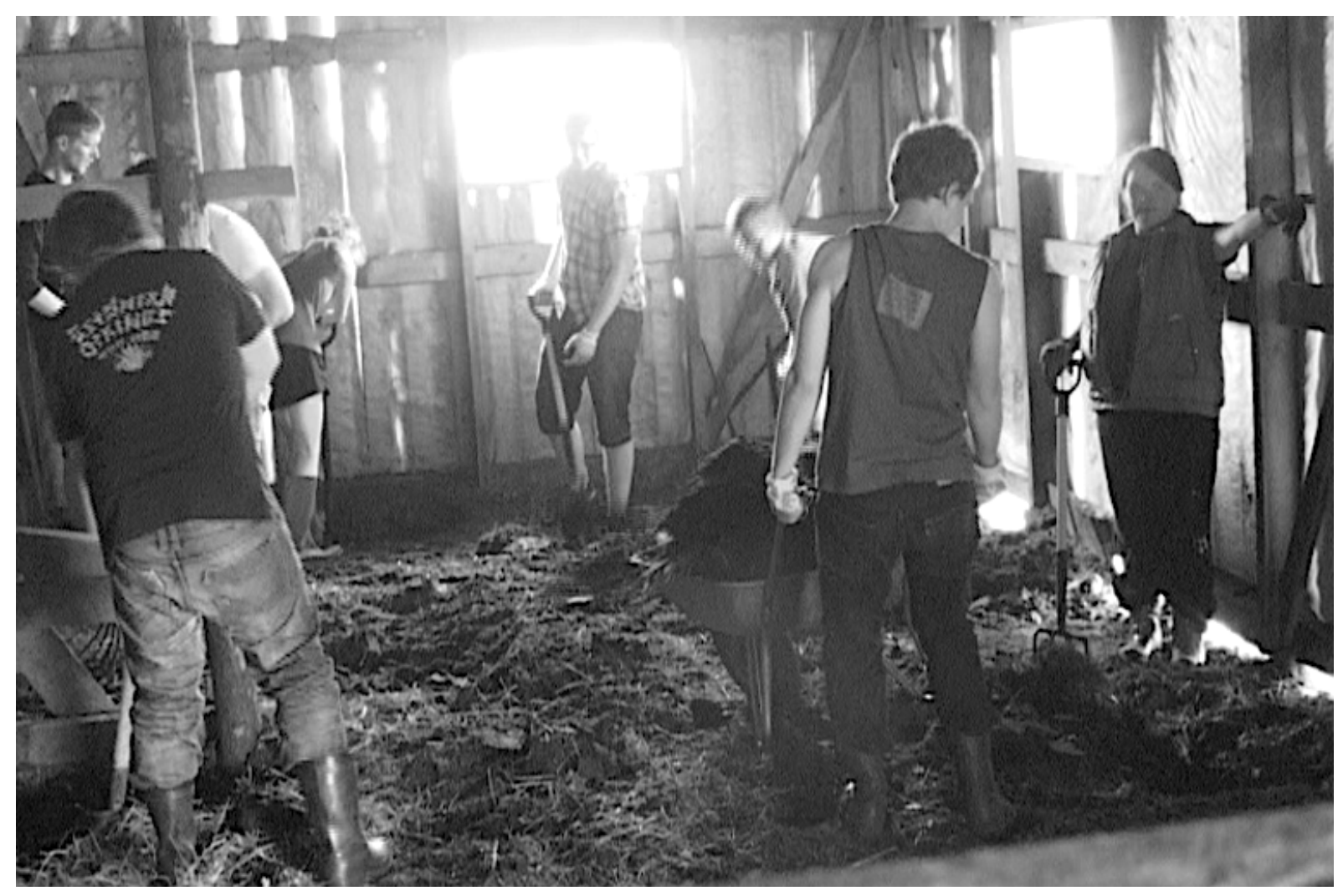

Fig. 5. Line (40): Vello dragging the wheelbarrow and Mari pointing.

Importantly, Mari's lifted hand is in position already during Vello's turn (as shown in line 39), which means that she uses the gesture for taking the turn, a major concern in organizing a conversation. Thus, the transient orders of the body and talk orient to the double activity frameworks, and transitions between the two may be seamless (in contrast, for the simultaneous deployment of the bodily recources in different trajectories of action, see Deppermann 2014). However, this moment of Mari disengaging from work also illustrates how the two activities begin to interfere with each other by requiring the same bodily resources. As studies on multiactivity have shown, this kind of situation may even lead to the impossibility of progressing the activities simultaneously (Haddington 2014a). Every participant's conduct is indicative 
of her preferential orientations to one activity being more relevant, necessary or urgent than another (Haddington 2014b:22-23). There is no work-related relevance for Mari's turn in lines (41-42). Within the conversational activity she is pursuing her own mock argument, disregarding Vello's oppositional move. Sequentially, it is important for her turn to be placed at the earliest possible moment before others can align with Vello, and the accompanying gesture is partly what legitimizes her timeout from the primary activity of work.

There are more and less legitimate moments for disengaging from work in order to talk. In the very first line of excerpt (1), Vello was waiting for the wheelbarrow to be filled, which was an organizationally defined pause. Also Mari in line (41) is fitting her withdrawal from work to the leaving wheelbarrow. At other times there are clear restrictions to involving the body in conversation, such as displaying recipiency. The work task entails heavy bodily engagement, either rolling the wheelbarrow in and out of the stable, or shoveling manure. While involved in these tasks (as shown in Figure 1), the participants are as a rule gazing at the surface or the lifted forkfuls. As a result, gaze is not always easily available for talk-related actions. In addition, bodily orientation in space is basically defined by the work requirements, such as the current frontline between the manure and the ground, and the related optimal positions for cutting off pieces of dung.

Importantly, however, the participants are bodily oriented toward the middle of the work area every time they throw a forkful of dung into the wheelbarrow. The wheelbarrow thus establishes a circular participant formation, not unlike a huge dinner table. In this way, the working arrangements simultaneously provide for a natural common focus, which should ease engagement in talk, should an occasion arise.

\section{Conclusion}

The paper focused on a single conversational sequence, and dissected it in terms of the two parallel participation frameworks: talk and physical labor. On the whole, there seems to be a tolerance of silence here that is almost unparalleled in talk-only contexts. Previously, Mushin and Gardner (2009) have presented examples of relatively long silences from a leisurly setting. Instead of residing on the more abstract notions of habits and culture, this paper argues that the extensive silences can be occasioned by the multiple moral concerns and layered participation of the potential speakers. Long between-turn silences are by-products of the simultaneous maintenance of the two activities. Crucially, it seems to be unproblematic for the participants in the current context to extend the temporal structure of a conversational sequence without having to reside to different mechanisms of re-engaging to re-enter the conversation mode. Next actions are here built unproblematically after silences and the case presented above represents one type of conversation in the co-present setting of insipient talk.

In this regard, it is crucial how and where we collect our "conversational" data. It has been an ideal for a long time to capture "maximally informal, spontaneous, naturally occurring conversations, with 2-6 consenting participants", as exemplified in Stivers et al. (2009) paper on the timing of question-answer pairs. In their study a following note appears: "Participants were often engaged in additional activities (e.g., eating, drinking, or stringing beads). As long as the task was not determining the direction or structure of 
the conversation this was considered acceptable" (Stivers et al. 2009: 10591). This statement nicely illustrates how data are collected in settings where additional activities are minimally disruptive and do not place high demands on the speakers bodies. The current paper instead looked at an activity setting where the hard physical labor, and the morality tied to it, seems to occasion conversation with partially distinct qualities. In particular, conversational sequences occur with a systematically different timing, when the physical work task is a priority.

The two participation frameworks were further shown to be represented in two kinds of talk: task-related contributions and chatting. The first one could be produced in a way that is sequentially inconsequential to the other: after a task-related utterance a prior chatting sequence can be continued with no perturbation. Participants apparently keep track of their different strands of participation while constructing next turns, in a way that can be described as parallel activity regimes (cf. Mondada 2014). The insertability of task-related utterances reflects the priority of work moral as compared to the moral obligation to produce the next relevant turn at talk. However, as the work here does not require constant cognitive attention, the longer silences after several hours of digging and lifting could also be reflective of the sheer physical fatigue, also a legitimate workrelated concern. This is featured in the lazy but sustained commitment to the nonconsequential, albeit possibly amusing, chatting activity. Instead of stating that the speakers here constantly opt out of conversation, it seems more accurate to analyze their occasional next turns as opting in, with no much commitment beyond the current turn.

Consequently, we should be aware of activity types when considering especially earlier ethnographic and cultural research that describes long silences as being typical for some ethnic groups, such as the Saami and the Finns (Reisman 1974: 112-113, Lehtonen and Sajavaara 1985, Sajavaara and Lehtonen 1997), both closely related languages and cultures to Estonian. In fact, Lehtonen and Sajavaara (1985: 200) explicitly state that in Finland it is not considered necessary to be engaged in small talk while eating. It may indeed be the case that different activities, such as eating, are treated as inhibiting talk to a different degree in different cultures. However, we need empirical records of the speakers' possible alternative preoccupations during the observations about the timing of talk. For example, it was not unusual for Estonian dialect collection to be carried out in collaborative work settings, where the researchers would join in to help out with urgent work tasks at the farms in order to be able to elicit dialect data from the farmers. Silences in that setting would probably have occurred quite similarly to the episode analyzed in the current paper, and affected the ethnographic observations.

When preoccupied with collaborative work, there seem to be legitimate ways of making oneself unavailable for next turn, displaying either inability to speak or disengagement, as described for different settings by Szymanski (1999) and Hoey (2015). Similarly to the above analysis, Hoey analyzes cases where the conclusion of talk and onset of silence are unremarkable and inconsequential events, as some other activity persists beyond it. In the current sheep stable setting, there are abundant resources available for the participants for not acting as a party in talk, such as shoveling, lifting, pulling, wiping the sweat etc. Conversation is nevertheless often kept going across longer sequences, and the speakers construct adjacency and nextness, albeit with some delay when compared to conversation-focused activities. They constantly have to negotiate the moral cost of 
potentially neglecting either the joint work task or the conversational sequence, often for the benefit of the first one.

\section{Transcription conventions}

\begin{tabular}{ll} 
underlining & - emphasis \\
\hline- & - truncation \\
{$[$ ] } & - overlaps; timing of embodied action \\
$=$ & - latching of turns \\
$(0.5)$ & - pause length in tenths of a second \\
$()$. & - micropause \\
$:$ & - lengthening of a sound \\
$<@>$ & - laughing quality \\
. $\mathrm{h}$ & - breathing in \\
$(\mathrm{m}) \mathrm{h}$ & - breathing out \\
0 & - low volume \\
boldface & - the focused particle and repeated materials in the excerpt \\
? & - pitch fall at the end of an intonation unit \\
$?$ & - pitch rise at the end of an intonation unit \\
, & - level pitch at the end of an intonation unit \\
- & - unfinished intonation unit \\
$\uparrow$ & - sharp rise in tone \\
$($ not in Estonian $)$ & - the part is not expressed in the Estonian version \\
$(\mathrm{X})$ & - unhearable syllable \\
$\#$ & - the moment of the frame grab \\
$*+$ & - the timing of embodied behavior \\
$>>>$ & - extension of the embodied behavior
\end{tabular}

References

Cekaite, Asta. 2015. "The Coordination of Talk and Touch in Adults Directives to Children: Touch and Social Control." Research on Language and Social Interaction 48(2): 152-175

Deppermann, Arnulf. 2014. "Multimodal Participation in Simultaneous Joint Projects: Interpersonal and Intrapersonal Coordination in Paramedic Emergency Drills." In Multiactivity in Social Interaction: Beyond Multitasking, ed. by P. Haddington, T. Keisanen, L. Mondada, and M. Nevile, 247-282. Amsterdam: Benjamins.

Egbert, Maria. 1997. "Schisming: The Collaborative Transformation from Single Conversation to Multiple Conversations." Research on Language and Social Interaction 30(1): 1-51.

Gardner, Rod, and Ilana Mushin. 2007. "Post-Start-Up Overlap and Disattentiveness in Talk in a Garrwa Community." Australian Review of Applied Linguistics 30(3): 35.1-35.14.

Goffman, Erving. 1963. Behavior in Public Places: Notes on the Social Organization of Gatherings. New York: Free Press.

Goffman, Erving. 1971. Relations in Public: Microstudies of the Public Order. New York: Basic Books. 
Goffman, Erving. 1981. Forms of Talk. Philadelphia: University of Pennsylvania Press. Goodwin, Charles. 1981. Conversational Organization: Interaction between Speakers and Hearers. New York: Academic Press.

Goodwin, Charles. 1984. "Notes on Story Structure and the Organization of Participation." In Structures of Social Action: Studies in Conversation Analysis, ed. by J. M. Atkinson, and J. Heritage, 225-246. Cambridge: Cambridge University Press. Goodwin, Charles. 2002. "Time in Action.” Current Anthropology 43: S19-S35.

Haddington, Pentti, Tiina Keisanen, Lorenza Mondada, and Maurice Nevile (eds). 2014a. Multiactivity in Social Interaction: Beyond Multitasking. Amsterdam: Benjamins.

Haddington, Pentti, Tiina Keisanen, Lorenza Mondada, and Maurice Nevile (eds). 2014b. "Towards Multiactivity as a Social and Interactional Phenomenon." In Multiactivity in Social Interaction: Beyond Multitasking, ed. by P. Haddington, T. Keisanen, L. Mondada, and M. Nevile, 3-32. Amsterdam: Benjamins.

Hoey, Elliott M. 2015. "Lapses: How People Arrive at, and Deal with, Discontinuities in Talk." Research on Language and Social Interaction 48(4):430-453.

Jefferson, Gail 1989. "Preliminary Notes on a Possible Metric which Provides for a 'Standard Maximum' Silence of Approximately One Second in Conversation." In Conversation: An Interdisciplinary Approach, ed. by P. Bull, and R. Derek, 166-196. Clevendon: Multilingual Matters.

Kasterpalu, Riina and Tiit Hennoste. 2016. "Estonian aa: A Multifunctional Change-ofState Token." Journal of Pragmatics 104: 148-162.

Keisanen, Tiina, Mirkka Rauniomaa, and Pentti Haddington. 2014. "Suspending a Course of Action: Managing Incompatibility in Moments of Multiactivity." In Multiactivity in Social Interaction: Beyond Multitasking, ed. by P. Haddington, T. Keisanen, L. Mondada, and M. Nevile, 109-134. Amsterdam: Benjamins.

Keevallik, Leelo. 2009. "The Grammar-Interaction Interface of Negative Questions in Estonian." SKY Journal of Linguistics 22, 139-173.

Lehtonen, Jaakko, and Kari Sajavaara. 1985. "The Silent Finn.” In Perspectives on Silence, ed. by D. Tannen, and M. Saville-Troike, 193-201. Norwood: Ablex.

Meyer, Christian. 2010. Self, Sequence and the Senses: Universal and Culture-specific Aspects of Conversational Organization in a Wolof Social Space.

Habilitationsschrift. Eingereicht an der Fakultät für Soziologie der Universität Bielefeld.

Mondada, Lorenza. 2011. "The Organization of Concurrent Courses of Action in Surgical Demonstrations." In Embodied interaction: Language and body in the material world, ed. by J. Streeck, C. Goodwin, and C. LeBaron, 207-226. Cambridge: Cambridge University Press.

Mondada, Lorenza. 2012. "Talking and Driving: Multi-Activity in the Car." Semiotica 191: $223-256$.

Mondada, Lorenza. 2014. "The Temporal Orders of Multiactivity: Operating and Demonstrating in the Surgical Theatre." In Multiactivity in Social Interaction: Beyond Multitasking, ed. by P. Haddington, T. Keisanen, L. Mondada, and M. Nevile, 33-75. Amsterdam: Benjamins.

Mushin, Ilana, and Rod Gardner 2009. "Silence is Talk: Conversational Silence in Australian Aboriginal Talk-in-Interaction." Journal of Pragmatics 41(10): 2033-2052.

Nevile, Maurice. 2015. "The Embodied Turn in Research on Language and Social Interaction." Research on Language and Social Interaction 48(2): 121-151. 
Raymond, Geoffrey, and Gene Lerner. 2014. "A Body and its Involvements: Adjusting Action for Dual Involvements." In Multiactivity in Social Interaction: Beyond Multitasking, ed. by P. Haddington, T. Keisanen, L. Mondada, and M. Nevile, 227-246. Amsterdam: Benjamins.

Reisman, Karl. 1974.. “Contrapuntal Conversations in an Antiguan Village.” In Explorations in the Ethnography of Speaking, ed. by R. Bauman, and J. Sherzer, 110-124. Cambridge: Cambridge University Press.

Sacks, Harvey. 1992. Lectures on Conversation [1964-1972] (Vol. 1 \& 2), ed. by G. Jefferson. Cambridge: Blackwell Publishers.

Sacks, Harvey, Emanuel A. Schegloff, and Gail Jefferson. 1974. "A Simplest Systematics for the Organization of Turn-taking for Conversation." Language 50(4): 696-735.

Sajavaara, Kari, and Jaakko Lehtonen. 1997. “The Silent Finn Revisited." In Silence. Interdisciplinary Perspectives, ed. by A. Jaworski, 263-283. Berlin: Mouton de Gruyter.

Schegloff, Emanuel A. 1998. "Body Torque." Social Research 65(3): 535-596.

Schegloff, Emanuel A., Gail Jefferson, and Harvey Sacks. 1977. "The Preference for SelfCorrection in the Organization of Repair in Conversation." Language 53(2): 361-382

Stivers, Tanja, Nick J. Enfield, Penelope Vello, Christina Englert, Makoto Hayashi, Trine Heinemann, Gertie Hoymann, Federico Rossano, Jan Peter de Ruiter, KyungEun Yoon, and Stephen C. Levinson. 2009. "Universals and Cultural Variation in Turn-taking in Interaction." Proceedings of the National Academy of Sciences 106(26): 10587-92.

Svennevig, Jan. 2008. "Trying the Easiest Solution First in Other-initiation of Repair." Journal of Pragmatics 40(2): 333-348.

Tannen, Deborah. 1985. “Silence: Anything But.” In Perspectives on Silence, ed. by D. Tannen, and M. Saville-Troike, 93-111. Norwood: Ablex.

Walsh, Michael. 1995. "Interactional Styles in the Courtroom: an Example from Northern Australia." In Language and the Law, ed. by J. Gibbons, 217-233. London: Longman. 